\title{
Malignant Female Reproductive System Neoplasm
}

National Cancer Institute

\section{Source}

National Cancer Institute. Malignant Female Reproductive System Neoplasm. NCI

Thesaurus. Code C4913.

A primary or metastatic malignant neoplasm involving the female reproductive system.

Representative examples include endometrial carcinoma, cervical carcinoma, ovarian

carcinoma, uterine corpus leiomyosarcoma, adenosarcoma, malignant mixed

mesodermal (mullerian) tumor, and gestational choriocarcinoma. 